\title{
Lung function in retired coke oven plant workers
}

\author{
N Chau, J P Bertrand, M Guenzi, L Mayer, D Téculescu, J M Mur, A Patris, J J Moulin, \\ Q T Pham
}

\begin{abstract}
Lung function was studied in 354 coke oven plant workers in the Lorraine collieries (Houillères du Bassin de Lorraine, France) who retired between 1963 and 1982 and were still alive on 1 January 1988. A spirometric examination was performed on $68 \cdot 4 \%$ of them in the occupational health service. Occupational exposure to respiratory hazards throughout their career was retraced for each subject. No adverse effect of occupational exposure on ventilatory function was found. Ventilatory function was, however negatively linked with smoking and with the presence of a respiratory symptom or discrete abnormalities visible on pulmonary $x$ ray films. The functional values were mostly slightly lower than predicted values and the most reduced index was the mean expiratory flow, $\mathbf{F E F}_{25-75 \%}$. The decrease in forced expiratory volume in one second $\left(F E V_{1}\right)$ was often parallel to that in forced vital capacity (FVC), but it was more pronounced for subjects who had worked underground, for smokers of more than 30 pack-years, and for subjects having a respiratory symptom. Pulmonary function indices were probably overestimated because of the exclusion of deceased subjects and the bias of the participants.
\end{abstract}

The Houillères du Bassin de Lorraine (HBL) (Lorraine collieries) have two coke oven plants, which started production in 1911 and 1955. The effect of occupational hazards on ventilatory function

INSERM U 115 "Santé au Travail et Santé Publique: Méthodes et Applications," Faculté de Médicine, BP 184, 54505 Vandoeuvre-les-Nancy Cedex, France N Chau, D Téculescu, J M Mur, A Patris, Q T Pham

Service de Médicine du Travail, Houillères du Bassin de Lorraine, Freyming-Merlebach

J P Bertrand, M Guenzi, L Mayer

Institut National de Recherche et de Sécurité (INRS), Vandoeuvre-les-Nancy

J M Mur, J J Moulin

Laboratoire d'Informatique Médicale, Faculté de Médicine, Vandoeuvre-les-Nancy

A Patris has been studied in this kind of industry and in other occupational sectors. ${ }^{1-16}$ Almost all of these studies, however, dealt with subjects who were still active (the studies by Bates $e t a l^{1}$ and Soutar et $a l^{1516}$ are exceptions), and there has been little research concerning retired workers. Therefore, we know little of the long term effect of occupational exposure on health, especially after retirement. Moreover, mortality from respiratory disease in the elderly may be linked to impairment of lung function. ${ }^{4}$

The aim of this work was to study the long term effect of occupational exposure on lung function in retired coke oven workers from the HBL. This work is part of a study on mortality and morbidity in survivors. ${ }^{17}$ It continues a previous study carried out in 1983 on mortality which showed an excess of mortality from lung cancer (standardised mortality ratio $(S M R)=2.51$ ) with reference to the French male population. ${ }^{18}$

\section{Material and methods}

The study sample consisted of all the male workers from the two coke oven plants who had retired between 1 January 1963 and 31 December 1982 (536 subjects) and who were still alive on 1 January 1988 (354 subjects). They were born between 1902 and 1935. Executives were excluded from the study.

The subjects were asked to attend a medical examination at the firm's occupational health centre. The protocol included a standard questionnaire, conducted by the occupational physician, concerning diseases treated or followed up for more than five years (cardiovascular, respiratory, digestive, infectious, and psychiatric diseases, traumas, cancers, etc), the questionnaire for the study of chronic bronchitis and pulmonary emphysema of the European Steel and Coal Commission (ECSC) of the EC, ${ }^{19}$ and the notion of "regular overconsumption" of alcoholic drink. Because of low numbers we did not distinguish between smokers and ex-smokers. The respiratory symptoms taken into account were chronic bronchitis defined by a daily cough and phlegm for three consecutive months each year over a period of two years, wheezing or chest noises heard daily, day and night, and dyspnoea classified into five stages.

To be aware of the respiratory symptoms in the period nearing retirement we sought information 
about cough and phlegm (considered separately) observed for more than 10 years. For the subjects who participated in this study, the mean duration between the date of their retirement and the time of the study was 11.6 (SD 4.4) years.

The protocol also included an electrocardiogram at rest, pulmonary radiography, and lung function tests. The radiographs were read at random by two physicians who were qualified to read the pulmonary films according to the 1980 International Labour Office (ILO) international classification. The measurements of lung function consisted of three forced expiration tests with a Vitalograph spirometer. The measurements were made in a standing position with an oral tip. The forced vital capacity (FVC), the forced expired volume in one second $\left(\mathrm{FEV}_{1}\right)$, and the mean expiratory flow $\mathrm{FEF}_{25-75 \%}$ were calculated using the criteria of the American Thoracic Society. ${ }^{20}$ Theoretical or predicted values were calculated using equations proposed by the ESCC. ${ }^{21}$

\section{OCCUPATIONAL EXPOSURES}

For each subject, the job history was retraced to define his occupational exposure during his working life, notably his exposure to dust, gas, and fumes due to the coal coking process, which contain polycyclic aromatic hydrocarbons. ${ }^{22}{ }^{23}$ The reconstitution of job history involved not only the jobs held in the coke oven plants, but also other jobs in the Charbonnages de France Group and in other companies, especially underground work, work in thermal power stations, the carbochemical sector, etc. It was compiled from the company's administrative files and occupational health files, and also with the cooperation of the coke oven plant foremen. The information thus gathered was confirmed by the subjects during their examination.

Each subject's job history was broken down into a succession of occupations for each of which were defined: the date of the beginning and the end of each occupation, the job itself, the work place (on or near the coke ovens, the washing plant, the workshops, underground, the thermal power station, etc) and the occupational nuisances-for example, the dust, gas and fumes produced by the coke ovens, coal dust, other particles, paint, welding, gas, smoke, benzol, phenol, sulphates, pitch, and tar.

The analysis of the jobs held during their careers showed that almost all of the subjects had one dominant job. This led us to define six types of homogenous exposure with each subject being classified in the exposure group that corresponded to the longest period of exposure when this period equalled or exceeded five years. The six were:

(1) Constant presence on the coke ovens (noted ovens): support setter, shield setter, oven regulator, door cleaner, column cleaner, and tar cleaning tube operator.

(2) Activity near the ovens and intermittent presence on the ovens (noted $\mathrm{N}$ ovens): pusher machine operator, stamper, switch operator, coke car, maintenance personnel on the ovens, fitters.

(3) Exposure in repair and maintenance workshops (noted workshops).

(4) Exposure to byproducts (noted byprod) in the oven gas treatment sector: benzol, phenol, sulphates, pitch, tars, etc.

(5) Underground work in coalmines (noted underground);

(6) Non- or slightly exposed (noted non-exp): administrative personnel, timekeeper, maintenance personnel who did not come into direct contact with the ovens, coal mixing work, porters, etc.

\section{STATISTICAL METHODS USED}

The statistical analysis consisted mainly of comparing each exposed group with the non-exposed group. The effects of smoking and of respiratory symptoms were also studied. The comparison of the subject's age and body height was made using the method of analysis of variance for one factor. ${ }^{24}{ }^{25}$ Smoking was defined according to the number of pack-years, differentiating between three groups of smokersnamely, 1-20, 21-30, and >30 pack-years. The comparisons of smoking and of the presence of abnormalities visible on the pulmonary $x$ ray films between each exposed group and the non-exposed group were made using the $\chi^{2}$ independence test or Fisher's exact probability test. ${ }^{25}$

The method of analysis of variance was used to compare the values of ventilatory indices between each exposed group and the non-exposed group, between the non-smokers and each category of smokers, and between subjects who had and those who did not have a respiratory symptom. To take into account the subject's age and body height, the analyses also refer to the value of those indices expressed as a percentage of the theoretical or predicted value.

To estimate the share of various factors on the ventilatory indices, we used the multiple linear regression method with a step down procedure and calculated the multiple correlation coefficient $r$. For each lung function index, the comparison of the slope of the regression line according to age between occupational exposure groups and between the smoker groups was made using the method of analysis of covariance. ${ }^{24} 26$ In this analysis, smoking and dyspnoea were considered as quantitative variables. For the other respiratory symptoms, the presence of abnormalities on the chest $x$ ray films, and the occupational exposure groups, we have defined indicator variables with the value 0 or 1 .

All the statistical tests were conducted with a first 


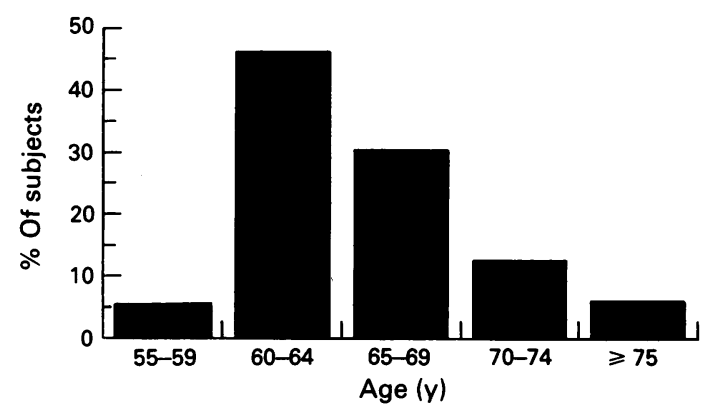

Histogram of age of subjects (mean age, 65.9 (SD 5.0) years).

order risk of $5 \%$ excluding the non-responses. The different statistical analyses were made using the Logist program. ${ }^{27}$

\section{Results}

Among the 354 subjects contacted $242(68.4 \%)$ attended a medical examination despite their age handicap that often made it necessary for them to be accompanied. The study sample finally included 231 subjects because 11 subjects were excluded due to lack of cooperation in the lung function tests.

Of the 112 subjects who did not participate in this study, 98 participated in the aforementioned enquiry on morbidity by completing a questionnaire sent by post. The number of subjects who refused to par- ticipate and those we lost contact with was small; five and nine subjects respectively. Subjects who had a spirometric examination were 2.3 years younger than the other subjects, but their occupational exposure and smoking were similar to subjects who did not undergo the examination; the prevalence of various diseases was also similar, except for diseases of the digestive system $(20.7 \% v 8.2 \%, p<0.01) .{ }^{17}$ Bias of the participants is therefore weak.

The figure shows that almost half of the subjects $(45.9 \%)$ were aged between 60 and 64 and about one third $(30.3 \%)$ between 65 and 69 . The mean age was 65.9 (SD 5.0). Table 1 shows the profile of different occupational exposure groups. Long exposure to several types of exposure was rare. The total duration of exposure to occupational hazards was 17.5 years for the underground group and about 23 years for the other exposed groups. It was only 1.4 years for the non-exposed group.

The age of non-exposed subjects (66.6 (SD 5.2) years) was close to that of each exposed group except the underground group (64.0 (SD 5.1) years; $p<0.05)$; this difference is explained by the fact that subjects who had worked underground retired earlier than subjects who had worked on the surface. The mean height was 1.68 (SD 0.07) $\mathrm{m}$. It was possible to determine smoking for $\mathbf{9 2 . 6 \%}$ of subjects; $\mathbf{9 0 . 7 \%}$ of them were smokers or ex-smokers and $55.6 \%$ smoked more than 20 pack-years. Smoking did not differ significantly between each exposed group and the non-exposed group.

Table 1 Profile of different exposure groups

\begin{tabular}{|c|c|c|c|c|c|c|c|c|c|c|}
\hline & \multicolumn{8}{|l|}{ Group } & \multirow{2}{*}{\multicolumn{2}{|c|}{ Total }} \\
\hline & Non-exp & Ovens & & Nover & & Workshops & Byprod & Underground & & \\
\hline No of subjects & 46 & 30 & & 67 & & 34 & 17 & 37 & 231 & \\
\hline Age (mean $(\mathrm{SD})(\mathrm{y}))$ & $66 \cdot 6 \quad(5 \cdot 2)$ & $65 \cdot 8$ & $(5 \cdot 7)$ & $65 \cdot 4$ & $(4 \cdot 1)$ & $67 \cdot 2 \quad(4 \cdot 9)$ & $67 \cdot 5 \quad(5 \cdot 1)$ & $64 \cdot 0(5 \cdot 1)^{\star}$ & $65 \cdot 9$ & $(5 \cdot 0)$ \\
\hline Height (mean $(\mathrm{SD})(\mathrm{m}))$ & $1.68(0.07)$ & 1.66 & $(0.06)$ & 1.69 & $(0.06)$ & $1.68(0.07)$ & $1.70(0.06)$ & $1.68(0.07)$ & 1.68 & $(0.07)$ \\
\hline $\begin{array}{l}\text { Smoking (No of pack-yea } \\
\text { Non-smokers } \\
1-20 \\
21-30 \\
>30 \\
\text { Unknown }\end{array}$ & $\begin{array}{l}\text { of subjects)): } \\
10 \cdot 9 \\
39 \cdot 1 \\
15 \cdot 2 \\
30 \cdot 4 \\
4 \cdot 3\end{array}$ & $\begin{array}{l}16 \cdot 7 \\
16 \cdot 7 \\
30 \cdot 0 \\
26 \cdot 7 \\
10 \cdot 0\end{array}$ & & $\begin{array}{r}6 \cdot 0 \\
32 \cdot 8 \\
16 \cdot 4 \\
37 \cdot 3 \\
7 \cdot 5\end{array}$ & & $\begin{array}{r}2.9 \\
41 \cdot 2 \\
14 \cdot 7 \\
35 \cdot 3 \\
5.9\end{array}$ & $\begin{array}{r}17 \cdot 7 \\
29 \cdot 4 \\
17 \cdot 6 \\
29 \cdot 4 \\
5 \cdot 9\end{array}$ & $\begin{array}{r}5 \cdot 4 \\
29 \cdot 7 \\
27 \cdot 0 \\
27 \cdot 0 \\
10 \cdot 8\end{array}$ & $\begin{array}{r}8 \cdot 7 \\
32 \cdot 5 \\
19 \cdot 5 \\
32 \cdot 0 \\
7 \cdot 4\end{array}$ & \\
\hline $\begin{array}{l}\text { Duration of different type } \\
\text { Ovens } \\
\text { Novens } \\
\text { Workshops } \\
\text { Byprod } \\
\text { Underground }\end{array}$ & $\begin{array}{cl}\text { exposure (mean } \\
0.1 & (0.6) \\
0.2 & (0.7) \\
0.2 & (0.7) \\
- & \\
0.9 & (1.5)\end{array}$ & $\begin{array}{l}\text { SD) }(y)) \\
13.5 \\
3.3 \\
2.8 \\
0.3 \\
3.9\end{array}$ & $\begin{array}{l}(6 \cdot 1) \\
(6 \cdot 1) \\
(6 \cdot 7) \\
(1 \cdot 0) \\
(7 \cdot 3)\end{array}$ & $\begin{array}{r}0.4 \\
18.0 \\
2 \cdot 7 \\
0.2 \\
2 \cdot 1\end{array}$ & $\begin{array}{l}(1 \cdot 1) \\
(7 \cdot 2) \\
(6 \cdot 6) \\
(1 \cdot 0) \\
(4 \cdot 6)\end{array}$ & $\begin{aligned} \overline{0} \cdot 2 & (0 \cdot 8) \\
21 \cdot 1 & (9 \cdot 7) \\
0 \cdot 7 & (2 \cdot 7) \\
1 \cdot 1 & (2 \cdot 2)\end{aligned}$ & $\begin{array}{rr}\bar{Z} & \\
2 \cdot 0 & (4 \cdot 7) \\
19 \cdot 1 & (7 \cdot 9) \\
2 \cdot 0 & (2 \cdot 8)\end{array}$ & $\begin{array}{cc}0 \cdot 2 & (0.7) \\
0 \cdot 2 & (1.0) \\
0 \cdot 4 & (1 \cdot 2) \\
0.04 & (0 \cdot 2) \\
16.6 & (6.4)\end{array}$ & $\begin{array}{l}1.9 \\
5.7 \\
4.5 \\
1.6 \\
4.3\end{array}$ & $\begin{array}{l}(5 \cdot 0) \\
(9 \cdot 1) \\
(9 \cdot 1) \\
(5 \cdot 5) \\
(7 \cdot 2)\end{array}$ \\
\hline All types combined & $1.4 \quad(1 \cdot 8)$ & $23 \cdot 8$ & $(8 \cdot 2)$ & $23 \cdot 4$ & $(7 \cdot 9)$ & $23 \cdot 1 \quad(9 \cdot 9)$ & $23 \cdot 1 \quad(9 \cdot 1)$ & $\cdot 17.5 \quad(6.6)$ & $18 \cdot 0$ & $(11 \cdot 3)$ \\
\hline Presence of discrete abno & $\begin{array}{l}\text { ties on the pulr } \\
27.3 \text { (33) }\end{array}$ & $\begin{array}{l}\text { nary } x \mathrm{r} \\
20.0\end{array}$ & $\begin{array}{l}\text { ray film } \\
\text { (15) }\end{array}$ & $\begin{array}{l}\% \text { of sut } \\
17.2\end{array}$ & $\begin{array}{l}\text { bjects): } † \\
(41)\end{array}$ & $22 \cdot 2(18)$ & $32 \cdot 0(25)$ & $14 \cdot 3(14)$ & $22 \cdot 6$ & (146) \\
\hline
\end{tabular}

†The examinations could only be conducted on 146 subjects $(63 \cdot 2 \%)$. The number of subjects who had a radiological examination is in parentheses. Comparison of means, comparison of frequencies, and homogeneity test between each exposed group and the non-exposed group (except for the duration of exposure where the difference is obvious).

${ }^{\star} \mathrm{p}<0.05$. 
Table 2 Values of ventilatory indices in relation to different risk factors

\begin{tabular}{|c|c|c|c|c|c|c|c|}
\hline Occupational exposure: & \multicolumn{2}{|c|}{$\begin{array}{l}F V C(l) \\
\text { Mean }(S D)\end{array}$} & \multicolumn{2}{|c|}{$\begin{array}{l}F E V_{1}(l) \\
\operatorname{Mean}(S D)\end{array}$} & $\begin{array}{l}F E V_{1} \mid F V C(\%) \\
\text { Mean }(S D)\end{array}$ & \multicolumn{2}{|c|}{$\begin{array}{l}F E F_{25-75 \%}(l / s) \\
\operatorname{Mean}(S D)\end{array}$} \\
\hline $\begin{array}{l}\text { Non-exp (46) } \\
\text { Ovens (30) } \\
\text { N ovens (67) } \\
\text { Workshops (34) } \\
\text { Byprod (17) } \\
\text { Underground (37) }\end{array}$ & $\begin{array}{l}3 \cdot 20 \\
3 \cdot 32 \\
3 \cdot 38 \\
3 \cdot 45 \\
3 \cdot 59 \\
3 \cdot 29\end{array}$ & $\begin{array}{l}(0.83) \\
(0.81) \\
(0.73) \\
(0.75) \\
(0.87) \\
(0.75)\end{array}$ & $\begin{array}{l}2 \cdot 32 \\
2 \cdot 46 \\
2 \cdot 47 \\
2 \cdot 64 \\
2 \cdot 68 \\
2 \cdot 37\end{array}$ & $\begin{array}{l}(0 \cdot 80) \\
(0 \cdot 72) \\
(0 \cdot 67) \\
(0 \cdot 69) \\
(0 \cdot 82) \\
(0 \cdot 65)\end{array}$ & $\begin{array}{l}70 \cdot 8(11 \cdot 5) \\
73.7(9 \cdot 0) \\
72.6(10.6) \\
76.4(7 \cdot 6)^{\star} \\
73.8(8 \cdot 0) \\
71.6(7.8)\end{array}$ & $\begin{array}{l}1 \cdot 79 \\
2 \cdot 19 \\
2 \cdot 11 \\
2 \cdot 31 \\
2 \cdot 30 \\
1 \cdot 93\end{array}$ & $\begin{array}{l}(0.93) \\
(1.06) \\
(1.07) \\
(1 \cdot 13)^{\star} \\
(1 \cdot 17) \\
(0.87)\end{array}$ \\
\hline $\begin{array}{l}\text { Smoking (No of pack-years): } \\
\text { Non-smokers (20) } \\
1-20(75) \\
21-30(45) \\
>30(74) \\
\text { Unknown (17) } \\
\text { Correlation coefficient } \dagger\end{array}$ & $\begin{array}{r}3 \cdot 45 \\
3 \cdot 45 \\
3 \cdot 33 \\
3 \cdot 18 \\
3 \cdot 53 \\
-0 \cdot 15\end{array}$ & $\begin{array}{l}(0.84) \\
(0.87) \\
(0.77) \\
(0.69) \\
(0.59)\end{array}$ & $\begin{array}{r}2 \cdot 61 \\
2 \cdot 60 \\
2 \cdot 39 \\
2 \cdot 26 \\
2 \cdot 78 \\
-0 \cdot 21\end{array}$ & $\begin{array}{l}(0.74) \\
(0.81) \\
(0.70) \\
(0.61)^{\star} \\
(0.56)\end{array}$ & $\begin{array}{cc}75 \cdot 1 & (8 \cdot 6) \\
74 \cdot 2 & (9 \cdot 3) \\
70 \cdot 8 & (11 \cdot 4) \\
70 \cdot 9 \quad(9 \cdot 5) \\
78 \cdot 5 \quad(5 \cdot 2) \\
-0.16\end{array}$ & $\begin{array}{r}2 \cdot 31 \\
2 \cdot 30 \\
2 \cdot 00 \\
1 \cdot 70 \\
2 \cdot 57 \\
-\mathbf{0 . 2 4}\end{array}$ & $\begin{array}{l}(1.18) \\
(1.21) \\
(0.96) \\
(0.76)^{\star \star} \\
(0.82)\end{array}$ \\
\hline \multicolumn{8}{|c|}{ Respiratory symptoms } \\
\hline $\begin{array}{l}\text { Cough: } \ddagger \\
\text { None }(203) \\
\geqslant 10 \text { years }(28)\end{array}$ & $\begin{array}{l}3.39 \\
3.02\end{array}$ & $\begin{array}{l}(0 \cdot 76) \\
(0 \cdot 88)^{\star}\end{array}$ & $\begin{array}{l}2 \cdot 51 \\
2 \cdot 12\end{array}$ & $\begin{array}{l}(0 \cdot 70) \\
(0 \cdot 82)^{\star \star}\end{array}$ & $\begin{array}{l}73 \cdot 5(9 \cdot 4) \\
68 \cdot 1(11 \cdot 2)^{\star \star}\end{array}$ & $\begin{array}{l}2 \cdot 13 \\
1 \cdot 64\end{array}$ & $\begin{array}{l}(1.04) \\
(0.97)^{\star}\end{array}$ \\
\hline $\begin{array}{l}\text { Phlegm: } \ddagger \\
\quad \text { None }(210) \\
\geqslant 10 \text { years }(21)\end{array}$ & $\begin{array}{l}3 \cdot 40 \\
2 \cdot 84\end{array}$ & $\begin{array}{l}(0 \cdot 77) \\
(0 \cdot 70)^{\star \star}\end{array}$ & $\begin{array}{r}2.51 \\
-\quad 1.98\end{array}$ & $\begin{array}{l}(0 \cdot 71) \\
(0 \cdot 71)^{\star \star}\end{array}$ & $\begin{array}{l}73 \cdot 4(9 \cdot 1) \\
67 \cdot 6(13 \cdot 6)^{\star \star}\end{array}$ & $\begin{array}{l}2.13 \\
1.53\end{array}$ & $\begin{array}{l}(1 \cdot 06) \\
(0 \cdot 74)^{\star}\end{array}$ \\
\hline $\begin{array}{l}\text { Wheezing: } \\
\text { None (185) } \\
\text { Day and night (46) }\end{array}$ & $\begin{array}{l}3.42 \\
3.07\end{array}$ & $\begin{array}{l}(0 \cdot 78) \\
(0 \cdot 72)^{\star \star}\end{array}$ & $\begin{array}{l}2 \cdot 54 \\
2 \cdot 14\end{array}$ & $\begin{array}{l}(0 \cdot 72) \\
(0 \cdot 65)^{\star \star \star}\end{array}$ & $\begin{array}{ll}73 \cdot 8 & (9 \cdot 6) \\
69 \cdot 2 & (9 \cdot 8)^{\star \star}\end{array}$ & $\begin{array}{l}2 \cdot 19 \\
1 \cdot 60\end{array}$ & $\begin{array}{l}(1.09) \\
(0.70)^{\star \star \star}\end{array}$ \\
\hline $\begin{array}{l}\text { Chronic bronchitis: } \\
\text { None/suspect (158) } \\
\text { Yes (70) }\end{array}$ & $\begin{array}{l}3.48 \\
3.07\end{array}$ & $\begin{array}{l}(0 \cdot 73) \\
(0 \cdot 84)^{\star \star \star}\end{array}$ & $\begin{array}{l}2 \cdot 61 \\
2 \cdot 15\end{array}$ & $\begin{array}{l}(0 \cdot 67) \\
(0 \cdot 75)^{\star \star \star}\end{array}$ & $\begin{array}{l}74 \cdot 6(8 \cdot 2) \\
69 \cdot 1(11 \cdot 9)^{\star \star \star}\end{array}$ & $\begin{array}{l}2 \cdot 26 \\
1 \cdot 67\end{array}$ & $\begin{array}{l}(1 \cdot 06) \\
(0 \cdot 89)^{\star \star \star}\end{array}$ \\
\hline $\begin{array}{l}\text { Dyspnoea at effort: } \\
\text { Stage } 0(107) \\
\text { Stage } 1(31) \\
\text { Stage } 2(70) \\
\text { Stage } \geqslant 3(23) \\
\quad \text { Correlation coefficient } \dagger\end{array}$ & $\begin{array}{r}3.55 \\
3.58 \\
3.09 \\
2.88 \\
-0.31\end{array}$ & $\begin{array}{l}(0.69) \\
(0.67) \\
(0 \cdot 83) \\
(0 \cdot 80)\end{array}$ & $\begin{array}{r}2 \cdot 70 \\
2 \cdot 66 \\
2 \cdot 22 \\
1.84 \\
-0.39\end{array}$ & $\begin{array}{l}(0 \cdot 67) \\
(0.55) \\
(0.70) \\
(0.68)\end{array}$ & $\begin{array}{cc}75 \cdot 7 & (8 \cdot 6) \\
74 \cdot 1 & (6 \cdot 1) \\
71 \cdot 3 & (9 \cdot 0) \\
63 \cdot 1 & (14 \cdot 0) \\
-0.37 & \end{array}$ & $\begin{array}{r}2.39 \\
2.18 \\
1.77 \\
1.34 \\
-0.34\end{array}$ & $\begin{array}{l}(1 \cdot 10) \\
(0.95) \\
(0.93) \\
(0.63)\end{array}$ \\
\hline $\begin{array}{l}\text { Presence of discrete abnorma } \\
\text { None (113) } \\
\text { Yes (33) }\end{array}$ & $\begin{array}{c}\text { on the pu } \\
3 \cdot 55 \\
3 \cdot 10\end{array}$ & $\begin{array}{l}\text { lmonary } x \mathrm{r} \\
(0.76) \\
(0.83)^{\star \star}\end{array}$ & $\begin{array}{l}2 \cdot 66 \\
2 \cdot 20\end{array}$ & $\begin{array}{l}(0.69) \\
(0.81)^{\star \star}\end{array}$ & $\begin{array}{l}74.6(8.5) \\
69.5(12.71)^{\star \star}\end{array}$ & $\begin{array}{l}2 \cdot 26 \\
1 \cdot 76\end{array}$ & $\begin{array}{l}(1.03) \\
(1.06)^{\star}\end{array}$ \\
\hline
\end{tabular}

The number of subjects is in parentheses.

Comparison of means between each exposed group and the non-exposed group, each group of smokers and the non-smokers, and subjects who had a respiratory symptom or discrete abnormalities on the pulmonary $x$ ray films with the other subjects, except for dyspnoea. $\star p<0.05 ; \star \star p<0.01 ; \star \star \star p<0.001$.

†Only values significantly different to zero are given.

$\ddagger$ For 10 years or more.

The presence of discrete abnormalities (interstitial syndrome type and accentuation of the frame) was noted on $22.6 \%$ of the 146 chest $x$ ray films taken, whereas $0 / 1$ density or pneumoconiotic images were not detected. The frequency of these abnormalities did not differ significantly between the non-exposed group and each exposed group.

The functional parameters can be analysed using values expressed as the percentage of the predicted values, but our analyses were made firstly on actual values because the predicted values were not generally determined on populations of the same age as the study population. Table 2 shows the value of pulmonary parameters in relation to occupational exposure, smoking, respiratory symptoms, and the presence of discrete abnormalities on the pulmonary $x$ ray films. Unexpectedly, the non-exposed subjects had the lowest values for all the indices (FVC, FEV $\mathrm{FEV}_{1} / \mathrm{FVC}$, and $\mathrm{FEF}_{25-75 \%}$ ), the difference being significant for subjects from the workshops and for $\mathrm{FEV}_{1} / \mathrm{FVC}$ and $\mathrm{FEF}_{25-75 \%}$ only.

The functional parameters decreased with smoking: the correlation coefficient was about $-0 \cdot 15$ for FVC and FEV $1 / F V C$, and -0.20 for $F_{1} V_{1}$ and FEF $_{25-75 \%}$; Non-smokers and smokers of 1 to 20 packyears had similar values, however. The difference was significant only between non-smokers and smokers of more than 30 pack-years for $\mathrm{FEV}_{1}$ and $\mathrm{FEF}_{25-75 \% \text {. }}$

Respiratory symptoms were frequent for those 
Table 3 Values FCV (\% pr), FEV, $(\% \mathrm{pr}), F E V_{1} / F C V(\% \mathrm{pr})$, and $F E F_{25-75 \%}(\%$ pr) according to different risk factors

\begin{tabular}{|c|c|c|c|c|}
\hline & $\begin{array}{l}F V C(\% p r) \\
\text { Mean }(S D)\end{array}$ & $\begin{array}{l}F E V_{1}(\% p r) \\
M e a n(S D)\end{array}$ & $\begin{array}{l}F E V_{1} / F V C(\% \text { pr }) \\
\operatorname{Mean}(S D)\end{array}$ & $\begin{array}{l}F E F_{25-75 \%}(\% \text { pr }) \\
\operatorname{Mean}(S D)\end{array}$ \\
\hline $\begin{array}{l}\text { Non-exp (46) } \\
\text { Ovens (30) } \\
\text { Novens (67) } \\
\text { Workshops (34) } \\
\text { Byprod (17) } \\
\text { Underground (37) }\end{array}$ & $\begin{array}{ll}86 & (21) \\
91 & (21) \\
87 & (16) \\
92 & (14) \\
93 & (22) \\
87 & (17)\end{array}$ & $\begin{array}{ll}85 & (28) \\
91 & (26) \\
86 & (21) \\
96 & (17) \\
94 & (25) \\
84 & (20)\end{array}$ & $\begin{aligned} 97 & (16) \\
100 & (12) \\
98 & (14) \\
104 & (10)^{\star} \\
101 & (11) \\
96 & (10)\end{aligned}$ & $\begin{array}{ll}71 & (37) \\
86 & (42) \\
77 & (38) \\
90 & (31)^{\star} \\
85 & (37) \\
71 & (29)\end{array}$ \\
\hline $\begin{array}{l}\text { Smoking (No of pack-years): } \\
\text { Non-smokers }(20) \\
1-20(75) \\
21-30(45) \\
>30(74) \\
\text { Unknown (17) } \\
\text { Correlation coefficient }+\end{array}$ & $\begin{array}{ll}93 & (20) \\
89 & (19) \\
90 & (20) \\
85 & (17) \\
92 & (14) \\
-0 \cdot 14\end{array}$ & $\begin{array}{ll}96 & (25) \\
91 & (25) \\
88 & (26) \\
82 & (20)^{\star} \\
97 & (18) \\
-0 \cdot 21 & \end{array}$ & $\begin{aligned} 103 & (12) \\
101 & (12) \\
96 & (15) \\
96 & (13)^{\star} \\
106 & (8) \\
-0 \cdot 17 & \end{aligned}$ & $\begin{array}{ll}89 & (38) \\
85 & (39) \\
77 & (39) \\
65 & (29))^{\star \star} \\
95 & (31) \\
-0 \cdot 24\end{array}$ \\
\hline \multicolumn{5}{|c|}{ Respiratory symptoms } \\
\hline $\begin{array}{l}\text { None }(203) \\
\geqslant 10 \text { years }(28)\end{array}$ & $\begin{array}{ll}89 & (18) \\
84 & (23)\end{array}$ & $\begin{array}{ll}89 & (22) \\
80 & (31)\end{array}$ & $\begin{aligned} 100 & (12) \\
93 & (15)^{\star \star}\end{aligned}$ & $\begin{array}{ll}80 & (36) \\
66 & (38)\end{array}$ \\
\hline $\begin{array}{l}\text { Phlegm: }:+ \\
\text { None }(210) \\
\geqslant 10 \text { years }(21)\end{array}$ & $\begin{array}{ll}89 & (18) \\
79 & (18)^{\star}\end{array}$ & $\begin{array}{ll}90 & (23) \\
75 & (25)^{\star \star}\end{array}$ & $\begin{aligned} 100 & (12) \\
92 & (18)^{\star \star}\end{aligned}$ & $\begin{array}{ll}80 & (37) \\
61 & (28)^{\star}\end{array}$ \\
\hline $\begin{array}{l}\text { Wheezing: } \\
\text { None (185) } \\
\text { Day and night (46) }\end{array}$ & $\begin{array}{ll}90 & (18) \\
83 & (17)^{\star}\end{array}$ & $\begin{array}{ll}91 & (23) \\
79 & (23)^{\star \star}\end{array}$ & $\begin{aligned} 100 & (13) \\
94 & (14)^{\star}\end{aligned}$ & $\begin{array}{ll}82 & (37) \\
65 & (31)^{\star \star}\end{array}$ \\
\hline $\begin{array}{l}\text { Chronic bronchitis: } \\
\text { None/suspect }(158) \\
\text { Yes }(70)\end{array}$ & $\begin{array}{ll}91 & (17) \\
84 & (20)^{\star \star}\end{array}$ & $\begin{array}{ll}92 & (21) \\
80 & (27)^{\star \star \star}\end{array}$ & $\begin{aligned} 101 & (11) \\
94 & (16)^{\star \star \star}\end{aligned}$ & $\begin{array}{ll}84 & (36) \\
68 & (37)^{\star \star}\end{array}$ \\
\hline $\begin{array}{l}\text { Dyspnoea at effort: } \\
\text { Stage } 0(107) \\
\text { Stage } 1(31) \\
\text { Stage } 2(70) \\
\text { Stage } \geqslant 3(23) \\
\text { Correlation coefficient } \dagger\end{array}$ & $\begin{array}{c}94(17) \\
92(14) \\
83(19) \\
73(17) \\
-0.36\end{array}$ & $\begin{array}{l}97(21) \\
92(18) \\
81(23) \\
63(20) \\
-0.44\end{array}$ & $\begin{array}{cc}103 & (12) \\
100 & (8) \\
97 & (12) \\
86 & (18) \\
-0.36 & \end{array}$ & $\begin{array}{ll}91 & (37) \\
80 & (35) \\
69 & (33) \\
48 & (19) \\
-0.37\end{array}$ \\
\hline $\begin{array}{l}\text { Presence of discrete abnormal } \\
\text { None (113) } \\
\text { Yes (33) }\end{array}$ & $\begin{array}{l}\text { e pulmonary } x \mathrm{r} \\
95 \quad(17) \\
\begin{array}{ll}81 & (20)^{\star \star \star}\end{array}\end{array}$ & $\begin{array}{ll}96 & (22) \\
78 & (28)^{\star \star \star}\end{array}$ & $\begin{aligned} 101 & (12) \\
94 & (17)^{\star \star}\end{aligned}$ & $\begin{array}{ll}86 & (35) \\
66 & (42)^{\star \star}\end{array}$ \\
\hline
\end{tabular}

The number of subjects is in parentheses; values significantly different from $100 \%$ are given in bold.

Comparison of means between each exposed group and the non-exposed group, each group of smokers and the non-smokers, and subjects who had a respiratory symptom or discrete abnormalities on the pulmonary $x$ ray films with the other subjects, except for dyspnoea. ${ }^{\star} p<0.05 ;{ }^{\star \star} p<0.01 ; \star \star \star \mathrm{p}<0.001$

tOni: vlues significantly different to zero are given.

${ }_{+}$For 10 years or more.

seen at the time of the medical examination (wheezing, chronic bronchitis, and dyspnoea stage $\geqslant 1$ ) and for those that appeared over 10 years (cough and phlegm)—that is, a period nearing retirement. ${ }^{17}$ The subjects who suffered from coughing, phlegm, wheezing, or chronic bronchitis had all functional values lower than those of the other subjects. In comparison with subjects who did not have any of the considered symptoms the decreases were $10 \%$ for $\mathrm{FVC}, 15 \%$ for $\mathrm{FEV}_{1}, 7 \%$ for $\mathrm{FEV}_{1} / \mathrm{FVC}$, and greater than $20 \%$ for $\mathrm{FEF}_{25-75 \%}$.

For dyspnoea, the value of these indices decreased progressively with the stage of the symptom; the correlation coefficients were approximately -0.30 for FVC and $\mathrm{FEF}_{25-75 \%}$, and -0.38 for $\mathrm{FEV}_{1}$ and $\mathrm{FEV}_{1} / \mathrm{FVC}$. The functional values of stage 1 subjects were none the less close to those for stage 0 subjects. Dyspnoea stage $\geqslant 2$, which concerned almost a third of the subjects, had a similar effect to that of coughing, wheezing, and chronic bronchitis. Subjects showing discrete abnormalities on the pulmonary $x$ ray films also had functional values significantly less than the other subjects.

Table 3 shows the results of the analysis of the values expressed as the percentage of the predicted values. The values of FVC (\% predicted (pr)), $\mathrm{FEV}_{1}$ $(\% \mathrm{pr})$, and $\mathrm{FEF}_{25-75 \%}(\% \mathrm{pr}$ ) were generally lower than $100 \%$. The non-exposed, $N$ ovens, and underground groups had the lowest values. The $\mathrm{FEF}_{25-75 \%}$ was the most reduced index. The $\mathrm{FEV}_{1}$ had a relative decrease often parallel to that of the FVC, but more pronounced for subjects who had worked under- 
Table 4 Equation of linear regression of ventilatory indices in relation to age, height, occupational exposure, smoking and respiratory symptoms

\begin{tabular}{|c|c|c|c|c|c|c|c|c|c|}
\hline \multirow[b]{2}{*}{ Explained variable } & \multicolumn{8}{|c|}{ Regression coefficients } & \multirow[b]{2}{*}{$r$} \\
\hline & $\operatorname{Age}(y)$ & $\begin{array}{l}\text { Height } \\
(m)\end{array}$ & Non-exp & $\begin{array}{l}\text { Work- } \\
\text { shops }\end{array}$ & Byprod & $\begin{array}{l}\text { Smoking } \\
\text { (pack-years) }\end{array}$ & Symptom & Constant & \\
\hline $\begin{array}{l}\text { FCV (1): } \\
\text { Asymptomatic subjects ( } 75) \\
\text { Symptoms not taken into account } \\
\text { Cough }(\geqslant 10 \text { years) } \\
\text { Phlegm ( } \geqslant 10 \text { years) } \\
\text { Wheezing } \\
\text { Chronic bronchitis } \\
\text { Dyspnoea at effort }\end{array}$ & $\begin{array}{c}(-0.030) \\
-0.044 \\
-0.044 \\
-0.039 \\
-0.038 \\
-0.035 \\
-0.041\end{array}$ & $\begin{array}{l}4 \cdot 5 \\
4 \cdot 7 \\
4 \cdot 7 \\
4 \cdot 7 \\
4 \cdot 8 \\
4 \cdot 7 \\
4 \cdot 8\end{array}$ & $\begin{array}{l}z \\
z \\
z \\
=\end{array}$ & $\begin{array}{l}\overline{(0 \cdot 24)} \\
(0 \cdot 24) \\
- \\
\overline{(0 \cdot 28)}\end{array}$ & $\begin{array}{l}\overline{(0.35)} \\
(0.35) \\
= \\
= \\
=\end{array}$ & $\begin{array}{c}(-0.0074) \\
-0.0061 \\
-0.0061 \\
-0.0066 \\
-0.0059 \\
-0.0058 \\
-0.0051\end{array}$ & $\begin{array}{l}- \\
-0.43 \\
-0.24 \\
-0.24 \\
-0.21\end{array}$ & $\begin{array}{l}-1.91 \\
-1.66 \\
-1.66 \\
-1.73 \\
-2.02 \\
-1.93 \\
-1.65\end{array}$ & $\begin{array}{l}0.52 \\
0.54 \\
0.54 \\
0.54 \\
0.53 \\
0.54 \\
0.61\end{array}$ \\
\hline $\begin{array}{l}\mathrm{FEV}_{1}(1): \\
\text { Asymptomatic subjects } \\
\text { Symptoms not taken into account } \\
\text { Cough }(\geqslant 10 \text { years }) \\
\text { Phlegm }(\geqslant 10 \text { years }) \\
\text { Wheezing } \\
\text { Chronic bronchitis } \\
\text { Dyspnoea at effort }\end{array}$ & $\begin{array}{l}-0.040 \\
-0.046 \\
-0.045 \\
-0.045 \\
-0.044 \\
-0.041 \\
-0.044\end{array}$ & $\begin{array}{l}4 \cdot 2 \\
3 \cdot 6 \\
3 \cdot 5 \\
3 \cdot 4 \\
3 \cdot 5 \\
3 \cdot 4 \\
3 \cdot 6\end{array}$ & $\begin{array}{l}= \\
\bar{z} \\
\bar{z}\end{array}$ & $\begin{array}{c}(0.27) \\
0.34 \\
0.32 \\
0.33 \\
0.32 \\
0.30 \\
(0.20)\end{array}$ & $\begin{array}{c}\overline{0} \\
0 \cdot 34 \\
0 \cdot 32 \\
(0 \cdot 30) \\
(0 \cdot 31) \\
(0 \cdot 28) \\
(0 \cdot 29)\end{array}$ & $\begin{array}{l}-0.0012 \\
-0.0081 \\
-0.0085 \\
-0.0086 \\
-0.0079 \\
-0.0078 \\
-0.0071\end{array}$ & $\begin{array}{l}(0.24) \\
-0.38 \\
-0.25 \\
-0.24 \\
-0.22\end{array}$ & $\begin{array}{l}-1.56 \\
-0.39 \\
-0.22 \\
-0.21 \\
-0.46 \\
-0.40 \\
-0.35\end{array}$ & $\begin{array}{l}0.63 \\
0.54 \\
0.55 \\
0.56 \\
0.56 \\
0.56 \\
0.64\end{array}$ \\
\hline $\begin{array}{l}\text { FEV } / \text { FCV }(\%): \\
\text { Asymptomatic subjects } \\
\text { Symptoms not taken into account } \\
\text { Cough }(\geqslant 10 \text { years) } \\
\text { Phlegm ( } \geqslant 10 \text { years }) \\
\text { Wheezing } \\
\text { Chronic bronchitis } \\
\text { Dyspnoea at effort }\end{array}$ & $\begin{array}{l}-0.46 \\
-0.45 \\
-0.43 \\
-0.43 \\
-0.42 \\
-0.37 \\
-0.42\end{array}$ & $\begin{array}{l}z \\
\bar{z} \\
\bar{z}\end{array}$ & $\begin{array}{l}= \\
\bar{z} \\
\bar{z}\end{array}$ & $\begin{array}{l}4 \cdot 0 \\
5 \cdot 3 \\
4 \cdot 9 \\
5 \cdot 1 \\
5 \cdot 0 \\
4 \cdot 7 \\
3 \cdot 6\end{array}$ & $\begin{array}{l}= \\
\bar{z} \\
\bar{z}\end{array}$ & $\begin{array}{l}-0.17 \\
-0.094 \\
-0.10 \\
-0.10 \\
-0.091 \\
-0.087 \\
-0.081\end{array}$ & $\begin{array}{c}-4.6 \\
-5 \cdot 2 \\
(-3.1) \\
-3.8 \\
-2.6\end{array}$ & $\begin{array}{r}109 \cdot 7 \\
103 \cdot 6 \\
103 \cdot 0 \\
103 \cdot 0 \\
102 \cdot 1 \\
99 \cdot 6 \\
104.9\end{array}$ & $\begin{array}{l}0.50 \\
0.32 \\
0.36 \\
0.36 \\
0.35 \\
0.37 \\
0.45\end{array}$ \\
\hline $\begin{array}{l}\text { FEF }_{25-75 \%}(1 / \mathrm{s}): \\
\text { Asymptomatic subjects } \\
\text { Symptoms not taken into account } \\
\text { Cough }(\geqslant 10 \text { years }) \\
\text { Phlegm ( }(10 \text { years }) \\
\text { Wheezing } \\
\text { Chronic bronchitis } \\
\text { Dyspnoea at effort }\end{array}$ & $\begin{array}{c}(-0.048) \\
-0.055 \\
-0.055 \\
-0.054 \\
-0.047 \\
-0.051 \\
-0.051\end{array}$ & $\begin{array}{l}6 \cdot 5 \\
3 \cdot 6 \\
3 \cdot 6 \\
3 \cdot 4 \\
3 \cdot 4 \\
3 \cdot 2 \\
3 \cdot 6\end{array}$ & $\begin{array}{l}- \\
-0.33 \\
-0.33 \\
(-0.32) \\
-0.40 \\
-0.43 \\
-0.35\end{array}$ & $\begin{array}{l}\overline{(0.34)} \\
(0.34) \\
(0.33) \\
= \\
=\end{array}$ & $\begin{array}{l}= \\
= \\
z \\
=\end{array}$ & $\begin{array}{l}-0.022 \\
-0.015 \\
-0.015 \\
-0.015 \\
-0.014 \\
-0.014 \\
-0.013\end{array}$ & $\begin{array}{l}- \\
-0.46 \\
-0.43 \\
-0.35 \\
-0.27\end{array}$ & $\begin{array}{l}-4.74 \\
- \\
-0.32 \\
- \\
0.53 \\
0.13\end{array}$ & $\begin{array}{l}0.58 \\
0.47 \\
0.47 \\
0.49 \\
0.49 \\
0.49 \\
0.55\end{array}$ \\
\hline
\end{tabular}

The analysis concerns 214 subjects. Only regression coefficients significantly different to zero are supplied: normal type $p<0.05$; italic type $p<0.01$; bold type $\mathrm{p}<0.001$; parentheses $0.05<\mathrm{p}<0.10$.

For all functional indices, when all symptoms are taken into account simultaneously, only dyspnoea has a regression coefficient significantly different from zero.

ground, smokers of more than 20 pack-years, and subjects having a respiratory symptom.

Because functional indices are related to age and height of subject and to the previous risk factors, the multiple regression method was used to estimate their respective role. A linear model with no interaction was chosen because the regression coefficient of age did not differ significantly between the nonexposed group and each exposed group, between all of the exposure groups, and between the smoker groups. Moreover, no significant interaction was found between occupational exposure and smoking (table 4). In table 4 we also give the value of the regression coefficients significantly different from zero at a risk of $10 \%$.

When we only considered age, height, occupational exposure, and smoking, the multiple correlation coefficient was about 0.50 for FVC, $\mathrm{FEV}_{1}$, and $\mathrm{FEF}_{25-75 \%}$, and 0.32 for $\mathrm{FEV}_{1} / \mathrm{FVC}$. The regression coefficients of age for FVC, FEV, $\mathrm{FEV}_{1} /$ FVC, and $\mathrm{FEF}_{25-75 \%}$ were respectively -0.044 ,
$-0.046,-0.45$, and -0.055 . These values were greater in absolute terms than those in ESCC formulae that define predicted values as:

FVC pr $(1)=-0.026$ age $(y)+5.757$ height $(\mathrm{m})$ $-4.345$

$\mathrm{FEV}_{1} \operatorname{pr}(1)=-0.029$ age $(\mathrm{y})+4.301$ height $(\mathrm{m})$ $+2.492$

$\mathrm{FEV}_{1} / \mathrm{FVC}$ pr $(\%)=-0.179$ age $(\mathrm{y})+87 \cdot 21$

$\mathrm{FEF}_{25-75 \%} \operatorname{pr}(1 / \mathrm{s})=-0.043$ age $(\mathrm{y})+1.944$ height $(\mathrm{m})-2.699$

The difference was only significant (unilateral test) for FVC (p < 0.05), FEV 1 (p < 0.02), and FEV 1 FVC ( $p<0.02)$. Asymptomatic subjects could be considered as a reference group; the regression coefficients of age also were higher in absolute value than those in ESCC formulae but the difference was not significant for any ventilatory indices.

Smoking had a significant effect on all functional values. For example, a smoker of 20 pack-years had a lower value compared with a non-smoker: 0.121 for FVC, 0.161 for $\mathrm{FEV}_{1}, \mathbf{1} \cdot \mathbf{9} \%$ for $\mathrm{FEV} / \mathrm{FVC}$, and 0.30 
Table 5 Equation of linear regression of ventilatory indices in relation to age, height, occupational exposure, smoking, and the presence of discrete abnormalities on the pulmonary $x$ ray films

\begin{tabular}{|c|c|c|c|c|c|c|c|c|}
\hline \multirow[b]{2}{*}{ Explained variable } & \multicolumn{7}{|c|}{ Regression coefficients } & \multirow[b]{2}{*}{$r$} \\
\hline & Age (y) & $\begin{array}{l}\text { Height } \\
(m)\end{array}$ & Workshops & Ovens & $\begin{array}{l}\text { Smoking } \\
\text { (pack-years) }\end{array}$ & $\begin{array}{l}\text { Radiological } \\
\text { abnormalities }\end{array}$ & Constant & \\
\hline $\begin{array}{l}\text { FCV (1) } \\
\text { FEV }_{1}(1) \\
\text { FEV }_{1} / \mathrm{FCV}(\%) \\
\mathrm{FEF}_{25-75 \%}(1 / \mathrm{s})\end{array}$ & $\begin{array}{l}- \\
\overline{(-0.034)}\end{array}$ & $\begin{array}{l}5 \cdot 5 \\
4.5 \\
\frac{-}{4 \cdot 6}\end{array}$ & $\begin{array}{l}(0 \cdot 36) \\
0.48 \\
6 \cdot 5 \\
0 \cdot 76\end{array}$ & $\begin{array}{l}- \\
-\end{array}$ & $\begin{array}{l}(-0.0063) \\
-0.0099 \\
-0.13 \\
-0.017\end{array}$ & $\begin{array}{l}-0.47 \\
-0.41 \\
-4 \cdot 7 \\
-0.40\end{array}$ & $\begin{array}{c}-5 \cdot 53 \\
-4 \cdot 67 \\
76 \cdot 3 \\
-2 \cdot 89\end{array}$ & $\begin{array}{l}0.56 \\
0.58 \\
0.38 \\
0.53\end{array}$ \\
\hline $\begin{array}{l}\text { FCV (\% pr) } \dagger \\
\text { FEV }_{1}(\% \text { pr }) \dagger \\
\text { FEV }_{1} / \mathrm{FCV}(\% \text { pr }) \dagger \\
\text { FEF }_{25-75 \%}(\% \text { pr }) \dagger\end{array}$ & & & $\begin{array}{c}9 \cdot 89 \\
16 \cdot 9 \\
8 \cdot 8 \\
30 \cdot 7\end{array}$ & $\begin{array}{l}\bar{z} \\
24 \cdot 0\end{array}$ & $\begin{array}{l}(-0.16) \\
-0.35 \\
-0.18 \\
-0.56\end{array}$ & $\begin{array}{r}-12 \cdot 1 \\
-15 \cdot 5 \\
-6 \cdot 5 \\
-15.5\end{array}$ & $\begin{array}{r}97 \cdot 0 \\
101 \cdot 1 \\
103 \cdot 4 \\
88 \cdot 7\end{array}$ & $\begin{array}{l}0.35 \\
0 \cdot 44 \\
0 \cdot 39 \\
0 \cdot 47\end{array}$ \\
\hline
\end{tabular}

The analysis concerns 132 subjects. Only regression coefficients significantly different from zero are supplied: normal type $p<0.05$; italic type $\mathrm{p}<0.01$; bold type $\mathrm{p}<0.001$; parentheses $0.05<\mathrm{p}<0.10$.

†In regression model, age and height are not naturally taken into account.

$1 / \mathrm{s}$ for $\mathrm{FEF}_{25-75 \%}$ In this study, no adverse effect of occupational exposure was seen.

When taking into account each respiratory symptom separately, coughing for more than 10 years only significantly influenced $\mathrm{FEV}_{1} / \mathrm{FVC}$ (a decrease of $4.6 \%$ ). Wheezing influenced FVC, $\mathrm{FEV}_{1}$, and $\mathrm{FEV}_{25-75 \%}$ with respective losses of $0.241,0.261$, and $0.43 \mathrm{l} / \mathrm{s}$. Chronic bronchitis had the equivalent effect of wheezing on FVC, $\mathrm{FEV}_{1}$, and $\mathrm{FEF}_{25-75 \%}$; however, it also influenced $\mathrm{FEV}_{1} / \mathrm{FVC}$ (loss of 3.8\%). Dyspnoea had an effect on all functional indices; each change in stage reduced the values of FVC, FEV , $\mathrm{FEV}_{1} / \mathrm{FVC}$, and $\mathrm{FEF}_{25-75 \%}$ respectively by $0.21 \mathrm{l}$, $0.221,2.6 \%$, and $0.27 \mathrm{l} / \mathrm{s}$. When taking into consideration all respiratory symptoms, only dyspnoea had a regression coefficient significantly different from zero; indeed most of the subjects with respiratory symptoms also had dyspnoea stage $\geqslant 1$.

The presence of discrete abnormalities on the pulmonary $x$ ray films was also associated with pulmonary function: the values of FVC, $\mathrm{FEV}_{1}$, $\mathrm{FEV}_{1} / \mathrm{FVC}$, and $\mathrm{FEF}_{25-75 \%}$ were reduced by $0.47 \mathrm{l}$, $0.411,4.7 \%$, and $0.401 / \mathrm{s}$ respectively (table 5 ).

The analysis of parameters as a percentage of the predicted values-namely, FVC (\%pr), FEV (\%pr), $\mathrm{FEV}_{1} / \mathrm{FVC}(\% \mathrm{pr})$, and $\mathrm{FEF}_{25-75 \%}$ (\%pr) (tables 5 and 6 ) gave results similar to those obtained with the actual values.

\section{Discussion}

This study required much effort to trace subjects who had left the company for several years; some had changed address or region, and some had returned to their native country. None the less, almost all the subjects were traced, thanks to direct collaboration between the Occupational Health Services, the companys' personnel management, the Regional Union for Miners' Social Security, the general practitioners, and the hospital physicians. In spite of the distance and the age handicap (often requiring the subject to be accompanied by a member of the family) $68.4 \%$ of the subjects contacted attended the medical examination. The reconstitution of each subject's job history enabled us to define his occupational exposure during his working life.

On the whole an effect of occupational exposure on ventilatory function was not found, but subjects from the workshops showed slightly better values than those from the other exposure groups. Unexpectedly, non-exposed subjects showed an $\mathrm{FEF}_{25-75 \%}$ 0.401 lower than the other groups (according to the regression model), but we did not find any differences for FVC, $\mathrm{FEV}_{1}$, and $\mathrm{FEV} / \mathrm{FVC}$. These results raise several hypotheses. The first is the healthy worker effect, ${ }^{121}$ which was noted by Soutar and Hurley ${ }^{15}$ in a study on coalminers and ex-coalminers. The second is due to the exclusion of dead subjects. Their frequency in relation to the original cohor ${ }^{18}$ varied between the exposure groups (non-exposed (43\%), ovens $(26 \%), \mathrm{N}$ ovens $(34 \%)$, workshops $(25 \%)$, byprod (43\%), underground ( $29 \%)$ ). The third is the bias of the participants, which should none the less be minimal because the prevalence of different diseases did not vary significantly between these subjects and the other subjects except for diseases of the digestive system and ischaemic cardiopathies. ${ }^{17}$ Moreover, the proportion of participants did not differ sigificantly between the non-exposed group $(69.6 \%)$ and each of the exposed groups (ovens $(71.7 \%), \mathrm{N}$ ovens $(64.8 \%)$, workshcps $(80.0 \%)$, byprod $(73.9 \%)$, underground $(76 \cdot 9 \%)$ ).

On the other hand, the subjects studied were exposed to atmospheric pollution at their workplace and sometimes at their home, which was often near the Carling or Marienau industrial platforms. ${ }^{28} \mathrm{An}$ epidemiological study on children between 9 and 12 years old in schools situated near the most exposed zone in comparison with children from nearby "non polluted" zones showed no significant difference in ventilatory function between the two groups, but a negative effect of parental smoking and coal fired 
Table 6 Equation of linear regression for $F C V(\% \mathrm{pr}), F E V_{1}(\% \mathrm{pr}), F E V_{1} / F C V(\% \mathrm{pr})$, and $F E F_{25-75_{\circ}}(\% \mathrm{pr})$ in relation to occupational exposure, smoking, and respiratory symptoms

\begin{tabular}{|c|c|c|c|c|c|}
\hline \multirow[b]{2}{*}{ Explained variable } & \multicolumn{4}{|c|}{ Regression coefficients } & \multirow[b]{2}{*}{$r$} \\
\hline & Workshops & $\begin{array}{l}\text { Smoking } \\
\text { (pack-years) }\end{array}$ & Symptom & Constant & \\
\hline $\begin{array}{l}\text { FCV (\% pr): } \\
\text { Asymptomatic subjects ( } 75) \\
\text { Symptoms not taken into account } \\
\text { Cough }(\geqslant 10 \text { years) } \\
\text { Phlegm ( } \geqslant 10 \text { years) } \\
\text { Wheezing } \\
\text { Chronic bronchitis } \\
\text { Dyspnoea at effort }\end{array}$ & $\begin{array}{l}= \\
z \\
z \\
=\end{array}$ & $\begin{array}{c}(-0.19) \\
-0.15 \\
-0.15 \\
-0.16 \\
-0.14 \\
-0.14 \\
(-0.13)\end{array}$ & $\begin{array}{l}-11.5 \\
-6.9 \\
-6.7 \\
-5.8\end{array}$ & $\begin{array}{l}98 \cdot 8 \\
92 \cdot 1 \\
92 \cdot 1 \\
93 \cdot 5 \\
93 \cdot 4 \\
94 \cdot 0 \\
98 \cdot 0\end{array}$ & $\begin{array}{l}(0.20) \\
0.14 \\
0.14 \\
0.23 \\
0.21 \\
0.22 \\
0.39\end{array}$ \\
\hline $\begin{array}{l}\mathrm{FEV}_{1}(\% \mathrm{pr}): \\
\text { Asymptomatic subjects } \\
\text { Symptoms not taken into account } \\
\text { Cough }(\geqslant 10 \text { years }) \\
\text { Phlegm ( } \geqslant 10 \text { years }) \\
\text { Wheezing } \\
\text { Chronic bronchitis } \\
\text { Dyspnoea at effort }\end{array}$ & $\begin{array}{c}\overline{9} \\
9 \cdot 9 \\
9 \cdot 2 \\
9 \cdot 3 \\
9 \cdot 2 \\
(8 \cdot 7) \\
-\end{array}$ & $\begin{array}{l}-0.38 \\
-0.28 \\
-0.30 \\
-0.30 \\
-0.28 \\
-0.27 \\
-0.24\end{array}$ & $\begin{array}{c}(-8.7) \\
-15.5 \\
-9.7 \\
-9.7 \\
-8.6\end{array}$ & $\begin{array}{r}106 \cdot 9 \\
93 \cdot 4 \\
94 \cdot 9 \\
95 \cdot 3 \\
95 \cdot 3 \\
96 \cdot 3 \\
103 \cdot 4\end{array}$ & $\begin{array}{l}0.33 \\
0.25 \\
0.28 \\
0.32 \\
0 \cdot 30 \\
0.32 \\
0.48\end{array}$ \\
\hline $\begin{array}{l}\mathrm{FEV}_{1} / \mathrm{FCV}(\% \text { pr }): \\
\text { Asymptomatic subjects } \\
\text { Symptoms not taken into account } \\
\text { Cough }(\geqslant 10 \text { years }) \\
\text { Phlegm }(\geqslant 10 \text { years }) \\
\text { Wheezing } \\
\text { Chronic bronchitis } \\
\text { Dyspnoea at effort }\end{array}$ & $\begin{array}{c}5 \cdot 3 \\
7 \cdot 0 \\
6 \cdot 5 \\
6 \cdot 7 \\
7 \cdot 0 \\
6 \cdot 3 \\
(4 \cdot 7)\end{array}$ & $\begin{array}{l}-0.24 \\
-0.13 \\
-0.14 \\
-0.14 \\
-0.13 \\
-0.12 \\
-0.11\end{array}$ & $\begin{array}{r}-6.4 \\
-7.4 \\
(-4.0) \\
-5.0 \\
-3.5\end{array}$ & $\begin{array}{l}108 \cdot 0 \\
100 \cdot 7 \\
101 \cdot 8 \\
101 \cdot 6 \\
101 \cdot 5 \\
102 \cdot 2 \\
104 \cdot 5\end{array}$ & $\begin{array}{l}0.45 \\
0.25 \\
0.30 \\
0.30 \\
0 \cdot 28 \\
0.31 \\
0.40\end{array}$ \\
\hline $\begin{array}{l}\text { FEF }_{25-75 \%}(\% \text { pr }): \\
\text { Asymptomatic subjects } \\
\text { Symptoms not taken into account } \\
\text { Cough }(\geqslant 10 \text { years }) \\
\text { Phlegm }(\geqslant 10 \text { years }) \\
\text { Wheezing } \\
\text { Chronic bronchitis } \\
\text { Dyspnoea at effort }\end{array}$ & $\begin{array}{l}z \\
z \\
z \\
-\end{array}$ & $\begin{array}{l}-0.76 \\
-0.48 \\
-0.51 \\
-0.51 \\
-0.47 \\
-0.47 \\
-0.44\end{array}$ & $\begin{array}{l}-15.4 \\
-20.9 \\
-15.2 \\
-13.5 \\
-10.9\end{array}$ & $\begin{array}{r}112 \cdot 3 \\
89 \cdot 4 \\
92 \cdot 0 \\
92 \cdot 0 \\
92 \cdot 4 \\
93 \cdot 2 \\
100 \cdot 4\end{array}$ & $\begin{array}{l}0.39 \\
0.24 \\
0.27 \\
0.29 \\
0.29 \\
0.29 \\
0.42\end{array}$ \\
\hline
\end{tabular}

The analysis concerns 214 subjects. Only regression coefficients significantly different from zero are supplied: normal type $p<0.05$; italic type $\mathrm{p}<0.01$; bold type $\mathrm{p}<0.001$; parentheses $0.05<\mathrm{p}<0.10$.

For all functional indices, when all symptoms are taken into account simultaneously, only dyspnoea has a regression coefficient significantly different from zero.

central heating was found..$^{29}{ }^{30}$ The nature, the degree of pollution in the air, and the duration of exposure, however, differed between these children and the subjects studied.

The $\mathrm{FEV}_{1}$ had a relative decrease often similar to that of the FVC but was more pronounced in subjects who had worked underground, smokers of more than 30 pack-years, and subjects with a respiratory symptom or discrete abnormalities on the pulmonary $x$ ray films. The interpretation of a decrease of the $F_{E V}$ / FVC not taking account of a possible decrease in FVC can lead to errors because a normal value may conceal a simultaneous decrease of the FEV ${ }_{1}$ and FVC. ${ }^{1}$ The $\mathrm{FEF}_{25-75 \%}$ was the most reduced index. This phenomenon was also seen by Meyer-Bisch et $a l^{10}$ and by numerous other authors in farmers exposed notably to weedkillers, pesticides, and dust. ${ }^{31}$ This seems to confirm the hypothesis from Prefaut $e t a^{\beta^{2}}$ who think that in the course of obstructive diseases, the lesions are predominant in the small bronchi, which represent only a small fraction of the total bronchial resistance. The presence of a respiratory symptom or discrete radiological abnormalities were associated with the ventilatory indices. Such results have also been seen by other authors, ${ }^{70111522}$ who studied FVC and FEV in particular. Likewise, the role of smoking is indisputable for all the functional indices, ${ }^{1142133-40}$; we note moreover a dose relation effect.

For all the occupational exposure groups, the values of FVC, $\mathrm{FEV}_{1}$, and $\mathrm{FEF}_{25-75 \%}$ were reduced compared with the predicted values. The regression coefficients of age for FVC, $\mathrm{FEV}_{1}, \mathrm{FEV}_{1} / \mathrm{FVC}$, and $\mathrm{FEF}_{25-75 \%}$ were also more pronounced than their theoretical values given in the ESCC formulae, but the difference was only significant for FCV, $\mathrm{FEV}_{1}$, and $\mathrm{FEV}_{1} / \mathrm{FVC}$. This observation should be modulated, however, by the fact that the decrease could be accelerated by age. ${ }^{12} 214142$

So, we wondered if the theoretical values proposed by the ESCC were too high for the elderly. From among the large number of works quoted by 
Quanjer, ${ }^{21}$ except for an oversight on our part, only that of Ericsson and Irnell ${ }^{36}$ concerned a population ( 56 men aged between 51 and 71 years) of an age fairly similar to that of our population (56 to 81 years). For FVC and $\mathrm{FEV}_{1}$, these authors obtained -0.043 and -0.045 for the regression coefficients of age respectively. These values were almost identical to those in the present study $(-0.044$ and -0.046 respectively). For FEV $_{1} /$ FVC the value of the coefficient obtained by Ericsson and Irnell ${ }^{36}$ was intermediate between that of the ESCC $(-0.179)$ and our study $(-0.45)$.

For FEF $_{25-75 \%}$ the reference values are few. Préfaut et al $^{32}$ published mean values determined on a sample of 190 male subjects aged between 6 and 91 and in good health. Comparison with these values is problematic because in this population the subjects over 50 years were few, although smokers of more than 30 pack-years, and subjects with coughing or spitting for more than 10 years, wheezing, chronic bronchitis, or dyspnoea stage $\geqslant 3$ had values lower than the limits proposed.

Due to the lack of studies on populations of the same age, the conclusions should be prudent. Nevertheless we estimate that the study population had reduced functional values in relation to the normal values. Moreover, it is likely that the results presented are overestimated for the two reasons already mentioned. The first is due to the exclusion of dead subjects. Bates et al, ${ }^{1}$ in a longitudinal study on 397 coalminers from the same region, Lorraine, who became part of the study eight years before taking their retirement, had shown that the value of FVC and $F E V_{1}$ measured at the first examination were lower, and their annual decrease greater, in subsequently dead subjects than in living subjects; in smokers and non-smokers alike. The second reason is the bias of the participants. Although the frequency of different diseases in these subjects did not differ from that of the non-participants, the participants were 2.3 years younger, ${ }^{17}$ and we cannot assume that their lung function was similar. It is unlikely that this bias should greatly influence the comparative results between each exposed group and the non-exposed group as the proportion of participants in each exposure group in the original population ${ }^{18}$ was similar.

Because there are few studies concerning coke oven plants, we have enlarged the comparison to other occupational sectors. Firstly, for studies carried out in the same region of Lorraine (this eliminates the problems of different ethnic groups), Bates $e t a l^{1}$ found an annual decrease of 0.0401 for FVC and 0.0481 for FEV in non-smoking survivors during the period after their retirement (mean age at retirement 55.4 years). These were almost identical to those of the regression coefficients of age in the present study $(-0.044$ and -0.046 respectively). Also they found values of FVC and FEV inferior to their predicted value for all groups considered (alive or dead, smokers or non-smokers).

In a longitudinal study over five years of 871 iron ore miners aged between 35 and 55 years, Pham et $a l^{14}$ found a more pronounced decrease in pulmonary function in underground miners who were still active in comparison with those who had retired. Nevertheless, contrary to our study, the values of FVC and $\mathrm{FEV}_{1}$ were slightly superior to their predicted values.

Kauffmann $e t$ al $^{6}$ studied ageing over 12 years in 556 men, aged between 30 and 54 years at the beginning of the study (1960), who came from companies in the Paris region. They showed that after adjustment for age, smoking, and the FEV value, the annual decrease of the last was 0.0441 for subjects non-exposed or exposed to a slight concentration of dust or only to gas, around 0.0531 for subjects exposed to heat or a large concentration of dust, and 0.0601 for those exposed to heat and a high concentration of dust and gas. We found that the change with age of the $\mathrm{FEV}_{1}$ in the least exposed subjects was similar to the regression coefficient of age in our study $(-0.046$; the effects of occupational exposure and smoking being eliminated), despite the selection bias as previously mentioned.

Soutar and Hurley ${ }^{15}$ looked for a relation between dust exposure and respiratory function in $1867 \mathrm{coal}$ miners and 2192 ex-coal miners (the subjects were examined in 1950 and seen again 22 years later). These authors found an annual decrease of 0.0421 and 0.0411 for FVC and FEV 1 respectively. These values are similar to those of Bates $e t a l^{1}$ and the regression coefficients of age in our population. For $\mathrm{FEV}_{1} / \mathrm{FVC}$ Soutar and Hurley found an annual decrease of $0 \cdot 19 \%$ whereas in our subjects, the age regression coefficient was $-0.45 \%$. They found that smokers and ex-smokers had similar values. Soutar and Hurley ${ }^{15}$ also noted an association, as did Love and Miller, ${ }^{8}$ between exposure to dust and the decrease of the $\mathrm{FEV}_{1}$. They estimated a loss of FEV of 0.231 for an exposure of $300 \mathrm{gh} / \mathrm{m}^{3}$. In view of the importance of the effects of smoking and different diseases, the effect of occupational exposure can be concealed by these factors.

\section{Conclusion}

Although occupational exposure to respiratory hazards during their working life was retraced for each subject, no adverse effect of this exposure on ventilatory function was found. Smoking, the presence of a respiratory symptom, or discrete abnormalities on the pulmonary $x$ ray films were found to be negatively linked to ventilatory function. On the whole the function values were slightly reduced in comparison with the normal values. The $\mathrm{FEF}_{25-75 \%}$ was the most reduced index. The decrease 
in FEV 1 was often similar to that in FVC, but more pronounced for subjects who had worked underground, smokers of more than 30 pack-years, and subjects having a respiratory symptom. Ventilatory indices were probably overestimated because of the exclusion of deceased subjects and the bias of the participants.

1 Bates DV, Pham QT, Chau N, Pivoteau C, Dechoux J, Sadoul P. A longitudinal study of pulmonary function in coal miners in Lorraine, France. Am J Ind Med 1985;8:21-32.

2 Collins HPR, Dick JA, Bennett JG, Pern PO, Rickards MA, Thomas-DJ, et al. Irregularly shaped small shadows on chest radiographs, dust exposure, and lung function in coalworkers' pneumoconiosis. $\mathrm{Br} J$ Ind Med 1988;45:43-55.

3 Drummond L, Luck R, Afacan AS, Wilson HK. Biological monitoring of workers exposed to benzene in the coke oven industry. $B r J$ Ind Med 1988;45:256-61.

4 Ebi-Kryston KL. Respiratory symptoms and pulmonary function as predictors of 10-year mortality from respiratory disease, cardiovascular disease, and all causes in the whitehall study. J Clin Fpidemiol 1988;41:251-60.

5 Jorgensen HS, Kolmodin-Hedman B, St Jernberg N. Follow-up study of pulmonary function and respiratory tract symptoms in workers in a Swedish, iron ore mine. J Occup. Med 1988;30:953-8.

6 Kauffmann F, Drouet D, Lellouch J, Brille D. Occupational exposure and 12-year spirometric changes among Paris area workers. Br J Ind Med 1982;39:221-32.

7 Krzyzanowski M, Sherrill DL, and Lebowitz MD. Longitudinal analysis of the effects of acute lower respiratory illnesses on pulmonary function in an adult population. Am J Epidemiol 1990;131:412-22.

8 Love RG, Miller BG. Longitudinal study of lung function in coal-miners. Thorax 1982;37:193-7.

9 Maclaren WM, Hurley FJ, Collins HPR, Cowie AJ. Factors associated with the development of progressive massive fibrosis in British coalminers: a case-control study. $\mathrm{Br} J$ Ind Med 1989;46:597-607.

10 Meyer-Bisch C, Pham QT, Mur JM, Massin N, Moulin JJ, Teculescu $\mathrm{D}$, et al. Respiratory hazards in hard metal workers: a cross sectional study. Br J Ind Med 1989;46:302-9.

11 Muir DCF. Pulmonary function in miners working in British collieries: Epidemiological investigation by the National Coal Board. Bulletin Europeen de Physiopathologie respiratoire 1975;11:404-14.

12 Mur JM, Pham QT, Chau N, Teculescu D, Wil P, Henquel JC. Le vieillissement "physiologique" en 5 ans de la fonction pulmonaire de 140 mineurs de fer. Respiration 1987;52: 246-53.

13 Musk AW, Cotes JE, Bevan C, Campbell MJ. Relationship between type of simple nine year follow up study of subjects with small rounded opacities. Br J Ind Med 1981;38:313-20.

14 Pham QT, Mur JM, Teculescu D, Chau N, Gabiano $M$ Gaertner M, Henquel JC. A longitudinal study of symptoms and respiratory function tests in iron miners. European Journal of Respiratory Diseases 1986;69:346-54.

15 Soutar CA, Hurley JF. Relation between dust exposure and lung function in miners and ex-miners. $\mathrm{Br} J$ Ind Med 1986;43: 307-20.

16 Soutar CA, Maclaren WM, Annis R, Melville AWT. Quantitative relations between exposure to respirable coalmine dust and coalworkers' simple pneumoconiosis in men who have worked as miners but have left the coal industry. $\mathrm{Br} J$ Ind Med 1986;43:29-36.

17 Mayer L, Chau N, Bertrand JP, Guenzi M, Patris A, Pham QT, et al. Morbidity in retired coke oven plant workers. Am J Ind Med (submitted)

18 Bertrand JP, Chau N, Patris A, Mur JM, Pham QT, Moulin JJ, et al. Mortality due to respiratory cancers in the coke oven plants of the Lorraine coalmining industry (Houillères du Bassin de Lorraine). Br J Ind Med 1987;44:559-65.

19 Brille D, Bolt W, Greve LH, Minette A, Sartorelli E: Questionnaire de la Haute Autorité de la CECA pour l'étude de la bronchite et de l'emphysème pulmonaire. Commission des Communautés Européennes CECA, 1962.

20 ATS Statement: Snowbird workshop on standardization of spirometry. Am Rev Respir Dis 1989;6:215-20.

21 Quanjer $\mathrm{Ph} \mathrm{H}$. Standardized lung function testing. European Community for Coal and Steel. Bulletin Europeen de Physiopathologie respiratoire 1983;19 (suppl. 5):45-51.

22 Jongeneenlen FJ, Leeuwen FE, Oosterink S, Anzion RB, Loop F, Bos RP, Veen HG. Ambient and biological monitoring of coke oven workers: determinants of the internal dose of polycyclic aromatic hydrocarbons. $B r J$ Ind Med 1990;47: 454-61.

23 Keimig DG, Slymen DJ, White O. Occupational exposure to coke oven emissions from 1979-1983. Arch Environ Health 1986;41:363-7.

24 Armitage P, Berry G. Statistical methods in medical research. Oxford: Blackwell Scientific Publications, 1987, 559 pages.

25 Schwartz D. Méthodes statistiques à l'usage des médecins et des biologistes. Paris: Fammarion, 1963-1970.

26 Freund RJ, Minton PD. Regression Methods. A tool for data analysis, statistics: textbooks and monographs, Vol 30. New York: Marcel Dekker Inc, 1979.

27 Chau N, Patris A, Martin J, Kohler F, Lambert JP. Conception d'un logiciel de traitement et d'interrogations statistiques de données LOGIST. Int J Biomed Comput 1985;16:95-118.

28 Patris A, Chau N, Téculescu D, Marchand F, Aubry C, Pham QT, et al. La pollution atmosphérique dans la plate-forme industrielle de Carling en 1984. Pollution Atmosphérique 1987;116:373-81.

29 Aubry C, Téculescu D, Chau N, Viaggi MN, Pham QT, Manciaux M. Santé respiratoire des enfants et pollution atmosphérique. I. Symptồmes respiratoires. Rev Mal Respir 1989;6:215-20.

30 Téculescu D, Pham QT, Aubry C, Chau N, Viaggi MN, Henquel JC, Manciaux M. Santé respiratoire des enfants et pollution atmosphérique. II. Fonction ventilatoire. Rev $\mathrm{Mal}$ Respir 1989;6:221-8.

31 Bouchy 0 . Le risque respiratoire dans le milieu agricole. $A$ propos d'une enquête épidémiologique chez 755 exploitants agricoles et salariés de coopératives de Meuse. (Thèse de Doctorat de Médecine) Nancy: Université de Nancy, 1990.

32 Préfaut $\mathrm{Ch}$, Tcheriatchoukine J, Guerrero AJ, Moutou $H$, Chardon G. Débit maximum expiratoire $25-75 \%$ dans une population de non-fumeurs. Valeurs moyennes et limites inférieures de la normale. Bulletin Europeen de Physiopathologie respiratoire 1980;16:25-40.

33 Ashley F, Kannel WB, Sorlie PD, Masson R. Pulmonary function: relation to ageing, cigarette habit and mortality. The Framingham study. Ann Intern Med 1975;82:739-45.

34 Bande J, Clement J, Van de Woestijne KP. The influence of smoking habits and body weight on vital capacity and FEV male air force personnel: a longitudinal and cross-sectional analysis. Am Rev Respir Dis 1980;122:781-90.

35 Blackburn H, Taylor HL, Parlin RW, Kihlberg J, Keys A. Physical activity of occupation and cigarette smoking. Arch Environ Health 1965;10:312-22.

36 Ericsson P, Imell L. Spirometric studies of ventilatory capacity in elderly people. Acta Med Scand 1969;185:179-84.

37 Ferris BG Jr, Chen H, Murphy RLH Jr. Chronic nonspecific respiratory disease in Berlin, New Hampshire, 1967-1973. A further follow-up study. Am Rev Respir Dis 1976;113:475-85.

38 Fletcher C, Peto R, Tinker C, Speizer FE. The natural history of chronic bronchitis and emphysema. Oxford: Oxford University Press, 1976.

39 Hepper NGG, Fowler WS, Helmholz HF Jr. Relationship of height to lung volume in healthy men. Dis Chest 1960;37: 314-20.

40 Huhti E. Ventilatory function in healthy non-smokers and smokers. Scandinavian Journal of Respiratory Diseases 1976;48:149-55.

41 Cara $M$, Hentz $P$, Aide-mémoire of spirographic practice for examining ventilatory function, 2nd ed. Luxembourg: Commission of the European Communities, 1971. (Industrial Health and Medicine, Series No 11.)

42 Cole TJ. Linear and proportional regression models in the prediction of ventilatory function. Journal of the Royal Statistical Society (A) 1975;138:297-328.

Accepted 16 September 1991. 